\title{
In Situ Synchrotron X-ray Study of Ultrasound Cavitation and Its Effect on Solidification Microstructures
}

\author{
JIAWEI MI, DONGYUE TAN, and TUNG LIK LEE
}

Considerable progress has been made in studying the mechanism and effectiveness of using ultrasound waves to manipulate the solidification microstructures of metallic alloys. However, uncertainties remain in both the underlying physics of how microstructures evolve under ultrasonic waves, and the best technological approach to control the final microstructures and properties. We used the ultrafast synchrotron X-ray phase contrast imaging facility housed at the Advanced Photon Source, Argonne National Laboratory, US to study in situ the highly transient and dynamic interactions between the liquid metal and ultrasonic waves/bubbles. The dynamics of ultrasonic bubbles in liquid metal and their interactions with the solidifying phases in a transparent alloy were captured in situ. The experiments were complemented by the simulations of the acoustic pressure field, the pulsing of the bubbles, and the associated forces acting onto the solidifying dendrites. The study provides more quantitative understanding on how ultrasonic waves/bubbles influence the growth of dendritic grains and promote the grain multiplication effect for grain refinement.

DOI: $10.1007 / \mathrm{s} 11663-014-0256-\mathrm{z}$

(C) The Author(s) 2014. This article is published with open access at Springerlink.com

\begin{abstract}
APPLYING ultrasonic waves inside liquid media have been widely used in industry, e.g., in ultrasonic cleaning, sonochemistry, and medical treatment. In the past few decades, extensive laboratory results have demonstrated that applying ultrasound waves into solidifying liquid alloys can lead to the refinement of alloy microstructures. ${ }^{[1-3]}$ Although ultrasound-induced grain refinement has been shown effective in many metallic alloy systems, almost all previous research have interpreted the mechanism of grain refinement based on post-mortem microstructural characterization of the solidified alloys and empirical correlation, if any, between the measured grain size and the input ultrasonic power ${ }^{[4,5]}$ A very recent high-speed imaging study of ultrasonic treatment of a solidifying organic transparent alloy revealed that the shock wave emitted from imploding bubbles can fracture the growing dendrites, ${ }^{[6]}$ increasing the grain multiplication effect that leads to the enhancement of grain refinement. However, in situ and real time studies of the fundamentals of how the highly dynamic ultrasonic waves and the ultrasonic bubbles interact with the liquid metal, the semisolid and solid phases nucleated during solidification have not been reported mainly due to the difficulties in studying the bubble dynamics in the opaque liquid metal. In this paper, we report a number of in situ imaging studies of the dynamic behavior of ultrasonic bubbles in a Sn-13 wt pet Bi and a Bi-8 wt pet $\mathrm{Zn}$ alloy using the ultrafast X-ray phase contrast imaging (PCI) facility housed at the Advanced Photon Source (APS), Argonne National Laboratory, US. The real-time imaging studies
\end{abstract}

JIAWEI MI, Senior Lecturer, and DONGYUE TAN and TUNG LIK LEE, Ph.D. Students, are with the School of Engineering, University of Hull, East Yorkshire, HU6 7RX, U.K. Contact e-mail: j.mi@hull.ac.uk Manuscript submitted October 1, 2014.

Article published online December 11, 2014. are complemented by a numerical simulation of the bubble dynamics using the classical Gilmore model. ${ }^{[7]}$

The experiments were carried out at the beamline 32-ID-B of APS, and the detailed description of the experiment can be found in References 8, 9. The undulator gap was set to 14 to $18 \mathrm{~mm}$ with the X-ray energy of 5.017 to $7.758 \mathrm{keV}$. The transmitted X-rays were converted into visible light by a fast scintillator crystal, and the signals were projected 45 deg to a CCD camera (Photron, Inc.). Images were recorded with a spatial resolution of $1 \mu \mathrm{m} / \mathrm{pixel}$, and the fields of view depend on the acquisition rate due to the limited readout speed of camera. The image acquisition rate can reach up to 271,560 frames per second (fps), and it is the fastest X-ray imaging beamline so far in the world.

Sn-13 wt pet Bi and Bi-8 wt pet $\mathrm{Zn}$ alloys were used in the studies because of their low melting temperatures. The cyclic ultrasonic acoustic pressures introduced into the liquid metal are calculated using the Helmholtz equation (Eq. [1]) and finite element-based commercial software Comsol Multiphysics as detailed in References 8, 9

$$
\begin{gathered}
\frac{(\omega / C)^{2}}{\rho} P_{\mathrm{a}}+\nabla \cdot\left(\frac{1}{\rho} \nabla P_{\mathrm{a}}\right)=0 \\
P_{\mathrm{a}}=P_{\mathrm{A}} \cos (\omega t) \\
P_{\mathrm{A}}=\sqrt{2 \rho C W / A},
\end{gathered}
$$

where $\rho$ and $C$ are the density and sound speed of the liquid, respectively, $\omega=2 \pi f$ is the angular frequency and $f$ is the frequency of sound wave, $P_{\mathrm{a}}$ is the alternate pressure source with an amplitude of $P_{\mathrm{A}}$ at the sonotrode wave emitting surface, $W$ is the input ultrasound power $(20 \mathrm{~W}$ is used in the experiments and simulations), and $A$ is the area of the ultrasound wave emitting surface (the 2-mm- 
diameter tip has a wave emitting surface of $3.14 \times 10^{-6} \mathrm{~m}^{2}$ ).

The calculated pressures were then used in the classical Gilmore model ${ }^{[7]}$ to simulate the oscillation of the ultrasound bubbles (the changes of bubble radius under the cyclic pressure field), the velocity, and pressure at the bubble wall during quasi-steady state oscillation and those during the implosion of the ultrasonic bubbles

$$
\begin{gathered}
\left(1-\frac{\dot{R}}{C}\right) R \ddot{R}+\frac{3}{2} \dot{R}^{2}\left(1-\frac{\dot{R}}{3 C}\right)=\left(1+\frac{\dot{R}}{C}\right) H \\
+\frac{R \dot{R}}{C}\left(1-\frac{\dot{R}}{C}\right) \frac{\mathrm{d} H}{\mathrm{~d} R} \\
C^{2}=\frac{n(P+B)}{\rho}\left(\frac{P+B}{P_{0}+B}\right)^{-\frac{1}{n}} \\
H=\frac{n}{n-1} \frac{\left(P_{0}+B\right)^{\frac{1}{n}}}{\rho}\left[(P+B)^{\frac{n-1}{n}}-\left(P_{\infty}+B\right)^{\frac{n-1}{n}}\right] \\
P=\left(P_{0}+\frac{2 \sigma}{R_{0}}\right)\left(\frac{R_{0}}{R}\right)^{3 \gamma}-\frac{2 \sigma}{R}-\frac{4 \mu \dot{R}}{R},
\end{gathered}
$$

where $R, P, H$, and $C$ are the radius, pressure at the bubble wall, enthalpy, and sound speed of the liquid, respectively; $R_{0}$ is the initial bubble radius, $\sigma$ and $\mu$ are the surface tension and viscosity of the liquid, $\gamma$ is the gas polytropic exponent, $B$ and $n$ are empirical constants that depend on the particular liquid, $P_{0}$ is the liquid ambient pressure at $1 \mathrm{~atm}$, and $P_{\infty}=P_{0}+P_{\mathrm{a}}$ is the pressure at infinite distance from the bubble.

The physical properties of the metallic alloys and other parameters used in the modeling are listed in Table I, and the calculation was made using Comsol Multiphysics.

Figure 1(a) shows a typical X-ray image acquired at $135,780 \mathrm{fps}$ with an input ultrasound power of $20 \mathrm{~W}$, and a group of ultrasonic bubbles was captured immediately below the sonotrode tip in liquid Sn-13 wt pet Bi alloy at $600 \mathrm{~K}\left(327^{\circ} \mathrm{C}\right)$. The diameters of the bubbles are in the range of 10 to $50 \mu \mathrm{m}$. Figure 1(b) shows a quasi-steady state bubble that oscillated at a position $\sim 2.3 \mathrm{~mm}$ away from the sonotrode tip in liquid Bi-8 wt pet $\mathrm{Zn}$ at $700 \mathrm{~K}\left(427^{\circ} \mathrm{C}\right)$. The images were processed to reveal the bubble contraction and expansion. The bubble radii were measured from the 10 images with a time interval of $0.185 \mathrm{~ms}$ between two consecutive images (an acquisition rate of $5413 \mathrm{fps}$ ). The measured radii were compared with the calculated radii from the Gilmore model coupled with the acoustic pressure amplitude of $0.105 \mathrm{MPa}$ at $2.3 \mathrm{~mm}$ away from the sonotrode tip. An initial bubble radius of $8 \mu \mathrm{m}$ based on experiment measurements was used. Figure 2(a) shows that the measured and modeled bubble radii agree well when the same time interval (0.185 ms) was used in the simulation. Figure 2(b) shows that the oscillation of the bubble inside the pressure field generates velocity at the bubble wall with a magnitude of 1 to $2 \mathrm{~ms}^{-1}$.

Figure 3 shows a series of images acquired at 135,780 fps in a liquid Sn-13 wt pet Bi alloy $(\sim 2.65 \mathrm{~mm}$ below the sonotrode tip) at $600 \mathrm{~K}\left(327^{\circ} \mathrm{C}\right)$ and an acoustic pressure amplitude of $0.5 \mathrm{MPa}$ (simulated), showing the dynamic expansion of the bubble and the sudden implosion of the bubble (the second image from the right in Figure 3(a)). The emitted pressure (shockwave as marked in Figure 3(a)) pushes the liquid away from the bubble wall, making a blur circle which is supposed to be the original bubble wall before collapse, and forming a bigger dark layer outside the blurred circle. At such a high acquisition rate $(7.36 \mu$ s between two consecutive images), we can capture 4.5 images in one wave period $(30 \mathrm{kHz})$, and one image indeed shows the characteristics of violent bubble implosion with shockwave emission. Apparently, the area affected by the shock wave is clearly reflected by the radius of mist layer which is $\sim 1 / 3$ larger than original bubble radius (radius of the bright area). By applying the simulated pressure amplitude, that is, $0.5 \mathrm{MPa}$ at the bubble position, into Gilmore model, the corresponding abrupt changes of the bubble radius and velocity at the bubble wall are

Table I. The Physical Properties of the Liquid Metals and the Glass Sample Holder for Modeling

\begin{tabular}{lcc}
\hline Liquid Metal & Bi-8 wt pct Zn $\left[700 \mathrm{~K}\left(427^{\circ} \mathrm{C}\right)\right]$ & Sn-13 wt pct Bi $\left[600 \mathrm{~K}\left(327{ }^{\circ} \mathrm{C}\right)\right]$ \\
\hline Density $(\rho)$ & $9561 \mathrm{~kg} \mathrm{~m}^{-3}$ & $7528 \mathrm{~kg} \mathrm{~m}^{-3}$ \\
Viscosity $(\mu)$ & $1.5 \times 10^{-3} \mathrm{~Pa} \mathrm{~s}^{[13]}$ & $0.5 \mathrm{~N} \mathrm{~m}^{-1} \mathrm{~Pa} \mathrm{~m}^{[13]}$ \\
Surface tension $(\sigma)$ & $0.376 \mathrm{~N} \mathrm{~m}^{-1[1]}$ & $2730 \mathrm{~m} \mathrm{~s}^{-1 *}$ \\
Ambient sound speed in liquid $(C)$ & $1790 \mathrm{~m} \mathrm{~s}^{-1 *}$ & \\
Speed of sound in borosilicate tube $\left(c^{*}\right)$ & $5300 \mathrm{~m} \mathrm{~s}^{-1}$ & \\
Density of borosilicate tube $\left(\rho^{*}\right)$ & $2.23 \times 10^{3} \mathrm{~kg} \mathrm{~m}^{-3}$ \\
Frequency of ultrasound $(f)$ & $30 \mathrm{kHz}$ & \\
Ultrasound power $(W)$ & $20 \mathrm{~W}$ & \\
Diameter of the sonotrode tip $(d)$ & $2 \times 10^{-3} \mathrm{~m}$ \\
Gas polytropic exponent $(\gamma)$ & 1.4 & $3046 \mathrm{bar}^{*[15]}$ \\
Empirical constant $(B)$ & $7.025^{*[15]}$ & \\
Empirical constant $(n)$ & &
\end{tabular}

*Due to lack of data, the speeds of sound in pure $\mathrm{Bi}$ and $\mathrm{Sn}$ were assumed for $\mathrm{Bi}-8$ wt pet $\mathrm{Zn}$ and $\mathrm{Sn}-13$ wt pet Bi, respectively. The values of empirical constants, $B$ and $n$, for water were used. 


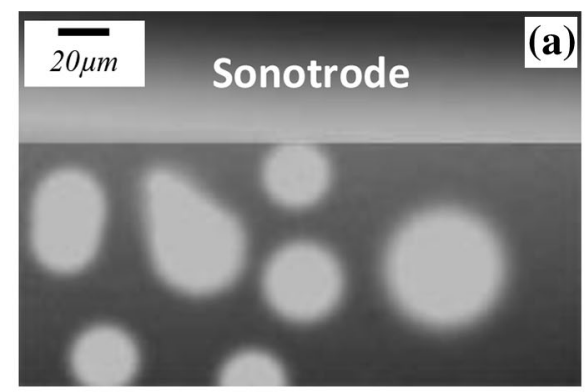

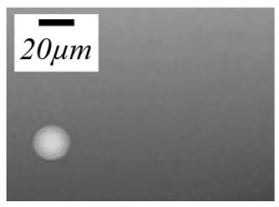

0

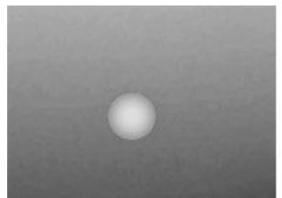

924

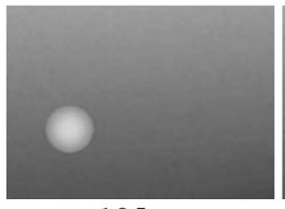

185

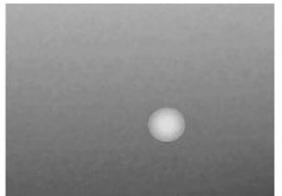

1108

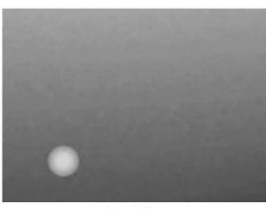

369

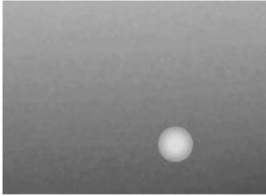

1293

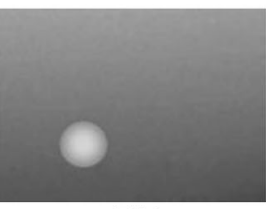

554

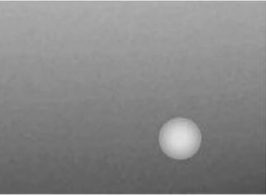

1478

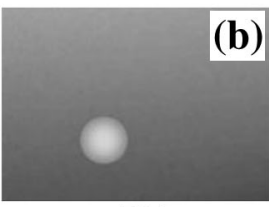

739

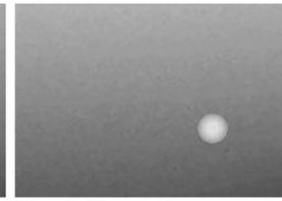

$1663(\mu \mathrm{s})$

Fig. 1-(a) A typical X-ray image acquired at 135,780 fps, showing a group of ultrasonic bubbles immediately below the sonotrode tip in a Sn-13wt pet Bi alloy $\left[600 \mathrm{~K}\left(327^{\circ} \mathrm{C}\right)\right]$, and $(b)$ the oscillation of a single bubble in liquid Bi-8wt pct $\mathrm{Zn}$ alloy $\left[700 \mathrm{~K}\left(427{ }^{\circ} \mathrm{C}\right)\right] \sim 2.3 \mathrm{~mm}$ below the sonotrode $(5413 \mathrm{fps})$, showing the quasi-steady state bubble oscillation under an acoustic pressure of $0.105 \mathrm{MPa}$.

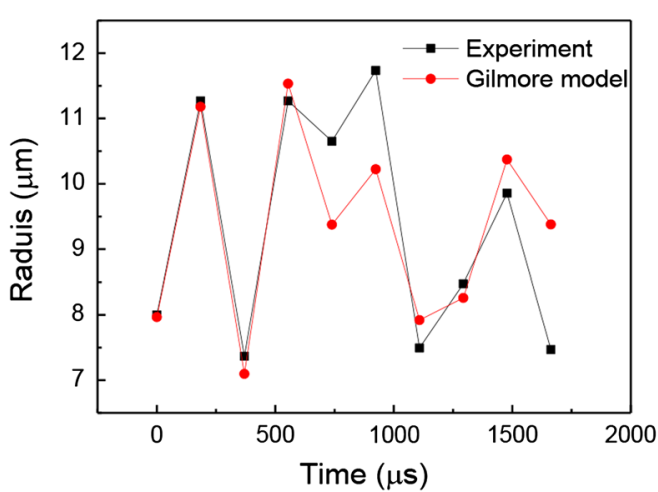

(a)

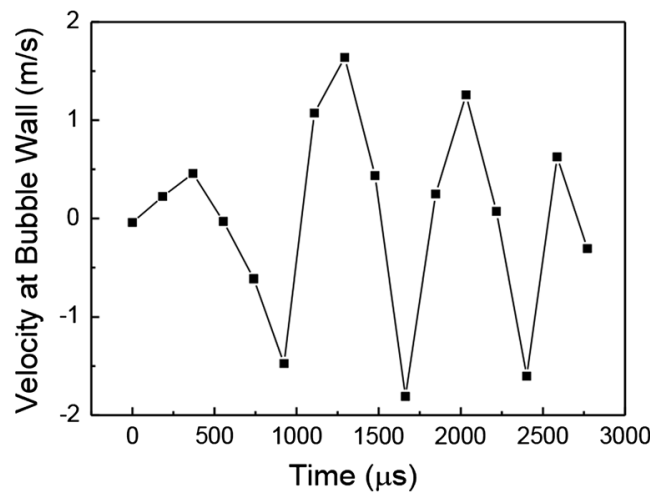

(b)

Fig. 2-(a) Bubble radii measured from the image sequence in Fig. 1(b) and comparison with the calculated bubble radii using Gilmore model (the initial radius $=8 \mu \mathrm{m}$, pressure amplitude $=0.105 \mathrm{MPa}$ ) and $(b)$ the velocities at the bubble wall calculated using the Gilmore model at same time step as the images showed in Fig. 1(b).

shown in Figures 3(b) and (c), respectively. Figure 3(b) shows that the measured radii agree well with the simulation, and Figure 3(c) shows that, at implosion, a shockwave (velocity jet) is emitted at the bubble wall, and the sudden shrink and rebounding of the bubble resulting in a velocity in the magnitude of 30 to $40 \mathrm{~ms}^{-1}$.

It is very difficult to capture the dynamic interactions between bubbles and the growing grains during solidification in the case of liquid metals because of the highly transient and dynamic process. A few attempts were made in the past using organic transparent alloy by Swallowe, ${ }^{[10]}$ and sucrose solutions by Chow et al. ${ }^{[11,12]}$ However, the relatively low image acquisition rate ( $500 \mathrm{fps}$ in Reference 10) used in those experiments cannot resolve the details of the highly dynamic inter- action between bubbles and dendrites. The author and his collaborators studied the similar phenomena in a transparent organic alloy at $8113 \mathrm{fps}$ as detailed in Reference 6 . Two typical images from ${ }^{[6]}$ are reproduced here to discuss the bubble interaction with the growing dendrites during the solidification process. Similar highspeed X-ray imaging studies of the interaction between bubbles and metallic grains have been just finished recently and will be reported elsewhere.

Figure 4 shows a quasi-steady state bubble of a diameter $\sim 0.2 \mathrm{~mm}$ oscillating or 'pulsating' at a primary dendrite tip. The quasi-steady state bubble just contacted the dendrite tip (Figure 4(a)). The bubble expanded and contracted periodically at the frequency of the imposed ultrasound, and created a "cleavage" at 

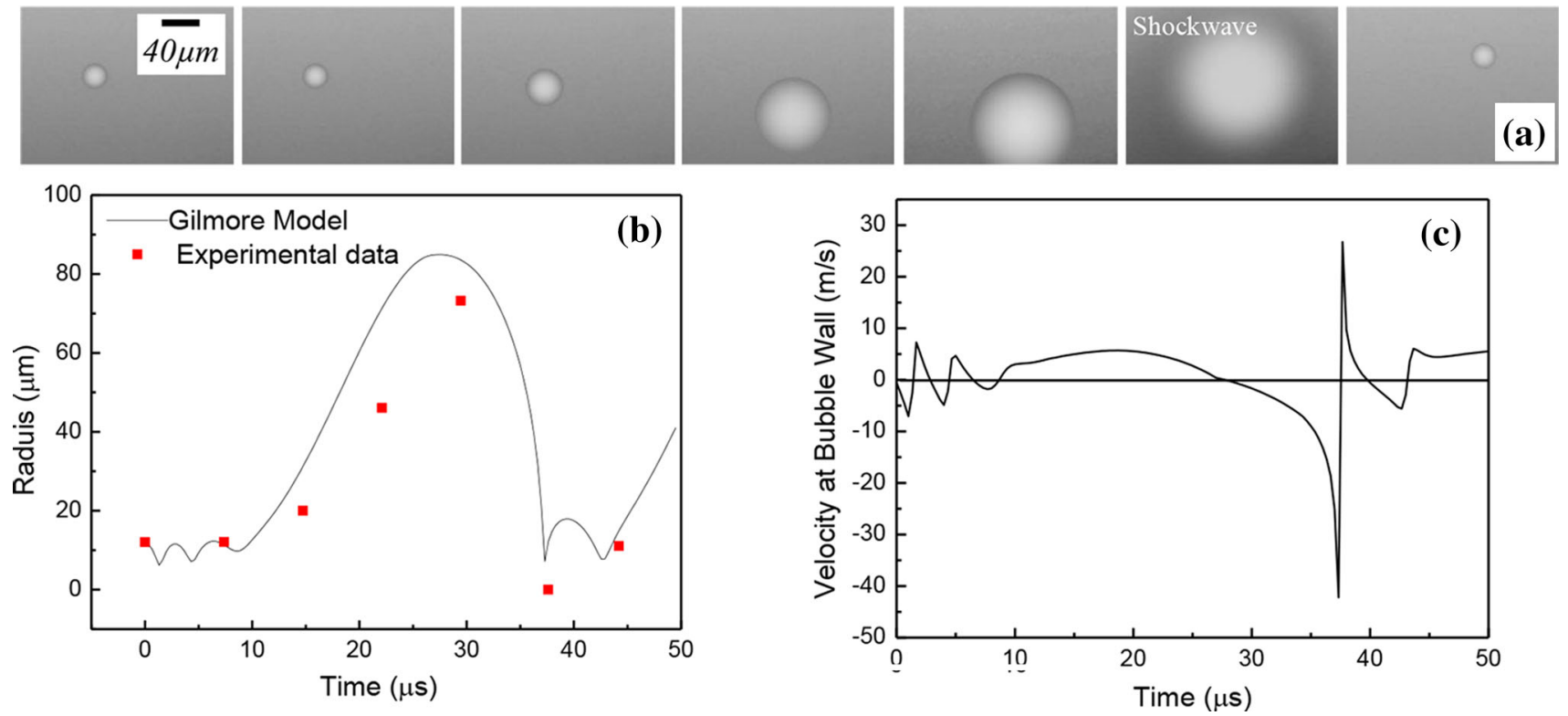

Fig. 3- (a) A series of images showing the expansion and implosion of a single bubble in a Sn- 13 wt pct Bi alloy [600 K (327 $\left.\left.{ }^{\circ} \mathrm{C}\right)\right]$ captured at $135,780 \mathrm{fps} \sim 2.65 \mathrm{~mm}$ below the sonotrode tip (time interval of $7.36 \mu \mathrm{s}$ ); $(b)$ the simulated abrupt change of the bubble radius before and at implosion, and $(c)$ the abrupt change of velocity at the bubble wall at implosion, emitting a shockwave.
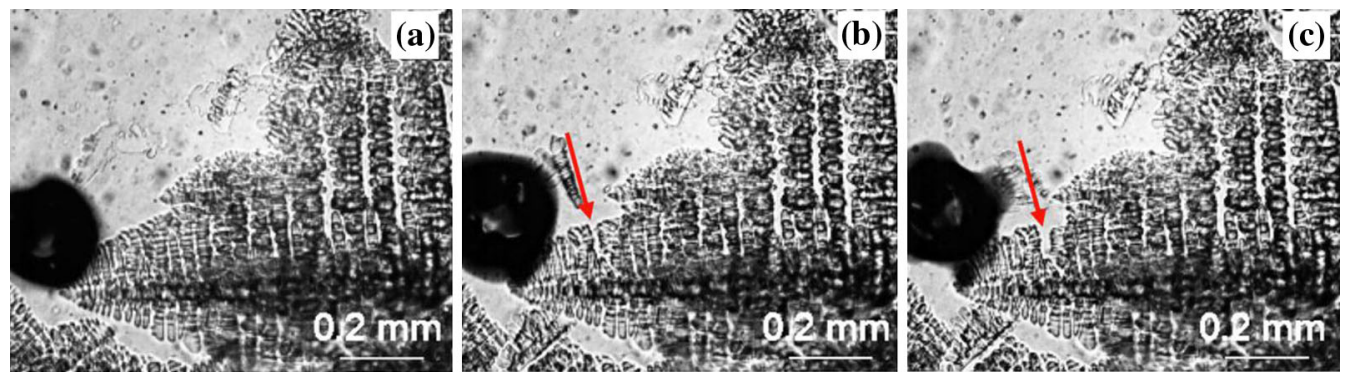

Fig. 4-The process of fracturing a secondary dendrite arm by a quasi-steady state bubble pulsing on the tip of a dendrite at $(a) t=0$, the start; (b) $t=13.93 \mathrm{~ms}$, after 279 periods; and $(c) t=15.77 \mathrm{~ms}$, after 315 periods. The images were recorded at $8,113 \mathrm{fps}^{[6]}$

a secondary dendrite arm after 279 wave periods $(13.93 \mathrm{~ms})$, and then as bending continued, the cleavage developed into partial fracture, breaking the dendrite side-arm from the trunk as shown in Figure 4(c). This whole sequence occurred over 315 wave periods, equivalent to $15.77 \mathrm{~ms}$.

Figure 5 shows three near-parallel dendrites nucleated on the relatively cold ultrasonic probe tip that grew approximately in a downward direction into the melt. Immediately after applying ultrasound, a single bubble became apparent in the melt which traveled rapidly toward the ultrasonic probe tip. It collided into a growing dendrite. Individual image frames were extracted from the video to highlight the motion of the bubble and its dendrite interaction. Figure 5(a) shows the instant when the bubble touched the dendrite tip. As the bubble moved upward in the direction indicated by the arrow in each subsequent image, the dendrite primary arm started to bend and a few secondary dendrite arms were detached progressively from the main trunk as shown in Figure 5(b). An outward shock wave caused by the expansion of bubble wall completely broke away secondary arms from the main trunk, as shown in Figure 5(c). The shock wave was characterized by a sudden halo of blurred, darker contrast around the bubble. Although the image acquisition rate was not high enough to resolve fully the details of bubble implosion, the sudden appearance of many dendrite fragments in images after the bubble implosion suggested that fragmentation of secondary dendrite arms occurred over a time scale much shorter than the interval of image acquisition of $0.44 \mathrm{~ms}$.

The ultrafast X-ray imaging is an ideal technique to study the highly transient phenomena of ultrasonic bubble dynamics in liquid metals. The oscillation and implosion of bubbles have been clearly captured and provided more in situ data for quantitative understanding on the relationship between bubble radius and the acoustic pressure field applied.

Experimental and simulation results indicate that the forces created by the oscillating bubbles and the shock 

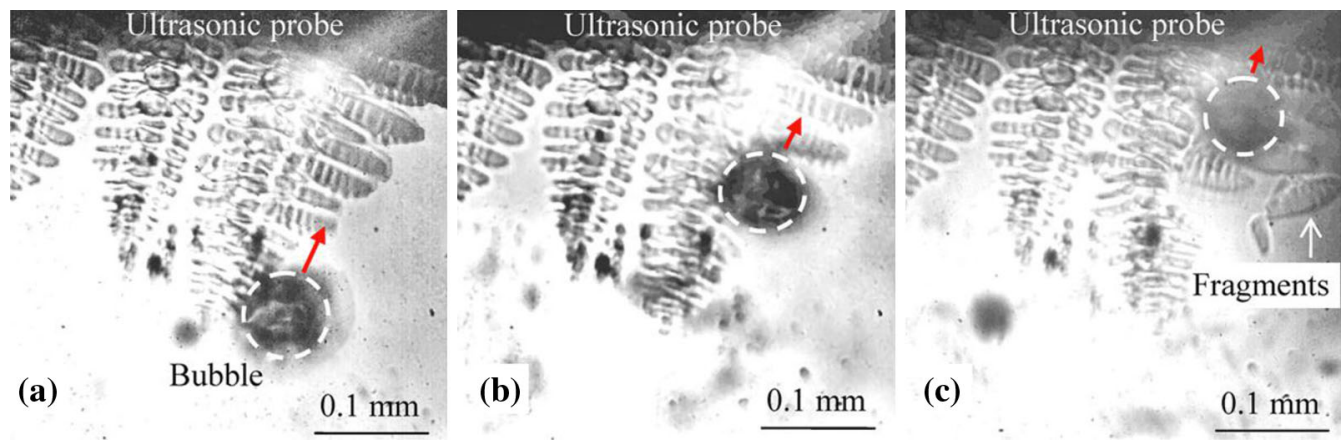

Fig. 5-High-speed images showing the interactions between a chaotic bubble (circled by dashed lines and the arrows pointing to the bubble trajectory) and a dendrite at bubble implosion. ${ }^{[6]}$

waves at bubble implosions are sufficient to fracture dendrite arms. This provides a solid evidence to support the theory that ultrasonic bubbles induced pulse or shock wave at implosion is one of the mechanisms to enhance grain multiplication effect and therefore promote grain refinement for metallic alloys during the solidification process.

The authors would like to acknowledge the financial support from the U.K. Engineering and Physical Sciences Research Council (Grant No. EP/L019965/1), The Royal Society Industry Fellowship (for J. Mi), Chinese Scholarship Council (for D.Y. Tan), and the award of the synchrotron X-ray beam time (GUP 23649 and GUP 26170) by the Advanced Photon Source, Argonne National Laboratory, US.

\section{OPEN ACCESS}

This article is distributed under the terms of the Creative Commons Attribution License which permits any use, distribution, and reproduction in any medium, provided the original author(s) and the source are credited.

\section{REFERENCES}

1. G.I. Eskin: Metallurgist, 2010, vol. 54 (7-8), pp. 505-13.

2. X. Jian, H. Xu, T.T. Meek, and Q. Han: Mater. Lett., 2005, vol. 59 (2-3), pp. 190-93.

3. X. Jian, T.T. Meek, and Q. Han: Scripta Mater., 2006, vol. 54, pp. 893-96.

4. T.V. Atamanenko, D.G. Eskin, L. Zhang, and L. Katgerman: Metall. Mater. Trans. A, 2010, vol. 41A, pp. 2056-66.

5. G.I. Eskin: Ultrasonic Treatment of Light Alloy Melts, $1^{\text {st }}$ ed., Gordon and Breach Science Publishers, Amsterdam, the Netherlands, 1998, pp 187-225.

6. D. Shu, B. Sun, J. Mi, and P.S. Grant: Metall. Mater. Trans. A, 2012, vol. 43A, pp. 3755-66.

7. F.R. Gilmore: Hydrodynamics Laboratory Report 26-4, California Institute of Technology, Pasadena, CA, 1952.

8. T.L. Lee, J.C. Khong, K. Fezzaa, and J. Mi: Mater. Sci. Forum, 2013, vol. 765, pp. 190-94.

9. D.Y. Tan and J. Mi: Mater. Sci. Forum, 2013, vol. 765, pp. 230-34.

10. G.M. Swallowe, J.E. Field, C.S. Rees, and A. Duckworth: Acta Mater., 1989, vol. 37, pp. 961-67.

11. R. Chow, R. Blindt, R. Chivers, and M. Povey: Ultrasonics, 2003, vol. 41 , pp. $595-604$.

12. R. Chow, R. Blindt, A. Kamp, P. Grocutt, and R. Chivers: Ultrason. Sonochem., 2004, vol. 11, pp. 245-50.

13. L.C. Prasad and R.K. Jha: Phys. Status Solid. (a), 2005, vol. 202(14), pp. 2709-19.

14. B.J. Keene: Int. Mater. Rev., 1993, vol. 38, pp. 157-92.

15. J. Holzfuss, M. Rüggeberg, and A. Billo: Phys. Rev. Lett., 1998, vol. 81 , pp. 5434-37. 